\title{
Unclassified Hepatocellular Adenoma
}

National Cancer Institute

\section{Source}

National Cancer Institute. Unclassified Hepatocellular Adenoma. NCI Thesaurus. Code C96761.

A hepatocellular adenoma without distinguished morphologic characteristics or known molecular abnormalities. 